\title{
Research Paper \\ The Effects of Topical Vitamin C on Burn Wound Healing
}

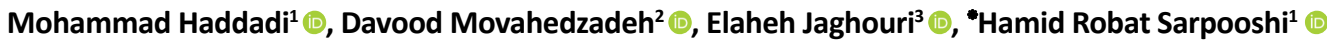

1. Department of Nursing, Faculty of Nursing and Midwifery, Sabzevar University of Medical Sciences, Sabzevar, Iran

2. Department of Health, Faculty of Nursing and Midwifery, Sabzevar University of Medical Sciences, Sabzevar, Iran.

3. Department of Nursing, Faculty of Nursing and Midwifery, Mashhad University of Medical Sciences, mashhad, Iran.

\begin{tabular}{|c|c|}
\hline $\begin{array}{l}\text { Use your device to scan } \\
\text { and read the article online }\end{array}$ & Citation: Haddadi M, Movahedzadeh D, Jaghouri E, Robat Sarpooshi H. [The Effect of Topical Vitamin C on Burn Wound \\
\hline 口itera & $\begin{array}{l}\text { Healing (Persian)]. Journal of Arak University of Medical Sciences (JAMS). 2021; 24(2):204-215. https://doi.org/10.32598/ } \\
\text { JAMS.24.2.6140.1 }\end{array}$ \\
\hline 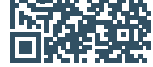 & doil'https://doi.org/10.32598/JAMS.24.2.6140.1 \\
\hline
\end{tabular}

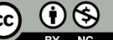

Article Info:

Received: 03 Apr 2020

Accepted: 27 Des 2020

Available Online: 01 Jun 2021

Keywords:

Vitamin C, Wound

healing, Burns,

Wound

\section{ABSTRACT}

Background and Aim Topical supplements and vitamins have often been used to accelerate the healing of burn wounds. This study investigated the effects of topical application of vitamin $\mathrm{C}$ solution on burn wounds.

Methods \& Materials This research was performed on 30 patients with extensive second-degree burns on one or two organs with similar burns. The study subjects were randomly divided into the intervention and control groups. The intervention group received topical application of vitamin $\mathrm{C}$ solution in addition to silver sulfadiazine ointment in the burn area; however, only silver sulfadiazine ointment was applied in the burn area in the control group. The wounds were examined and scored on days 1, 3, 7, and 14 using the Bates-Jensen Wound Assessment Tool. The collected data were analyzed in SPSS using repeatedmeasures Analysis of Variance (ANOVA).

Ethical Considerations This study was approved by the ethics committee of sabzevar university medical sciences. (Code: IR.MEDSAB.REC.1394.60).

Results Eighteen of the 30 examined patients were male and 12 were female. Their Mean $\pm S D$ age and body mass index were $43.03 \pm 11.90$ years and $27.82 \pm 4.08 \mathrm{~kg} / \mathrm{m}^{2}$, respectively.

Conclusion The repeated-measures ANOVA data indicated that the study groups were significantly different concerning the final mean scores given to the wounds $(P=0.047)$. In other words, the research groups significantly differed in wound healing; thus, topical application of vitamin $\mathrm{C}$ significantly improved wound healing in the study participants.

\section{Extended Abstract}

\section{Introduction}

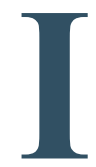

njuries caused by direct contact or contact with heat, chemical, electrical, or radiation sources are called burns [1]. Healing of burn wounds is among the problems of medical sciences [2]. In burns, the migration of keratinocytes is delayed by the accumulation of secretions and tissues on the wound surface; thus, the regeneration process of epithelial cells is postponed. Therefore, topical antibiotics must be used to control the infection in these wounds. Silver has been used for more than 50 years with bactericidal and bacteriostatic properties as a substance with excellent therapeutic effects on burn injuries. The adverse effects of silver sulfadiazine include pain, burning, itching, rash, and reversible leukopenia. Vitamin C can improve the immune system's functioning and provide wound healing. The current study aimed to evaluate the effects of topical vitamin $\mathrm{C}$ solution on burn wounds in patients with grade 2 burns.

\footnotetext{
* Corresponding Author:
}

Hamid Robat Sarpooshi

Address: Department of Nursing, Faculty of Nursing and Midwifery, Sabzevar University of Medical Sciences, Sabzevar, Iran.

Tel: +98 (514) 4217146

E-mail: sarpooshi92@mail.com 
Table 1. Demographic characteristics of the study subjects in quantitative indicators

\begin{tabular}{ccccc}
\hline Characteristic & Mean \pm SD & Max. & Min. & No. \\
\hline Age, $y$ & $43.03 \pm 11.90$ & 59.00 & 21.00 & 30 \\
\hline BMI, $\mathrm{kg} / \mathrm{m}^{2}$ & $27.82 \pm 4.08$ & 40.43 & 21.04 & 30 \\
\hline Sample's income & $99.33 \pm 2.19$ & 1500000 & 500000 & 30 \\
\hline Burn percentage & $7.96 \pm 5.64$ & 26 & 1 & 30 \\
\hline
\end{tabular}

\section{Materials and Methods}

In this study, 30 patients with grade 2 burns on two or more limbs symmetrically or one limb in a broad sense, in parallel, and one patient was randomly selected. Patients' wounds were randomly divided into intervention and control groups. Initially, the study patients' wounds were examined by an observer physician based on the Bates-Jensen Wound Assessment. In the limb or control area, on one limb or part of the wound, after routine washing and debridement with normal saline water and serum and dry with sterile gauze, $1 \%$ sulfadiazine silver ointment with sterile gloves up to $1.5-\mathrm{mm}$ thick was placed. In the opposite limb or other parts of the studied limb, as the infected limb or intervention area, after washing and debridement with normal saline water and serum, the wound was dried with sterile gauze; then, $10 \%$ vitamin $\mathrm{C}$ solution was applied to the wound surface and ointment was applied on it. Silver sulfadiazine 1\% was applied with sterile gloves with a thickness of $1.5 \mathrm{~mm}$. Finally, the burned wound was treated with Vaseline and dry gauzes, respectively.

\section{Results}

The study sample included 30 patients referring to the burn ward of Sabzevar Vasei Hospital; of them, 8 were male and 12 were female, with a Mean \pm SD age of $43.03 \pm 11.90$ and the BMI of $27.82 \pm 4.08 \mathrm{~kg} / \mathrm{m}^{2}$.

The results of repeated-measures ANOVA suggested that the mean final score of the wound between the two types of treatment was statistically significant $(\mathrm{P}=0.047)$, i.e., the rate of wound healing was significantly different between the two groups and topical vitamin $\mathrm{C}$ had a significant effect on the healed wounds.

Regarding the within-group effects, the impact of time on the mean final score of the wound was statistically significant; the mean final score of the wound on the first, third, seventh, and fourteenth days was significantly different $(\mathrm{P}=0.000, \mathrm{~F}=525.156)$, In other words, different days or times have affected the wound healing. The ANOVA test data concerning the interactive effect of wound coverage type and time identified that the overall wound score was

Table 3. The Mean \pm SD of final wound score in the intervention and control groups after bandage

\begin{tabular}{|c|c|c|c|c|}
\hline \multirow{2}{*}{\multicolumn{2}{|c|}{ Statistic }} & \multicolumn{3}{|c|}{ Mean $\pm S D$} \\
\hline & & Test & & Control \\
\hline \multirow{4}{*}{$\begin{array}{l}\text { Wound's total } \\
\text { score }\end{array}$} & Day 1 & $34.10 \pm 1.34$ & & $34.06 \pm 1.31$ \\
\hline & Day 3 & $35.50 \pm 4.73$ & & $35.73 \pm 4.73$ \\
\hline & Day 7 & $25.10 \pm 3.89$ & & $28.06 \pm 4.60$ \\
\hline & Day 14 & $18.63 \pm 3.14$ & & $22.10 \pm 4.46$ \\
\hline \multicolumn{2}{|c|}{ Time effect (within-group effect) } & $F=525.156$ & $d f=2.397$ & $P<0.001$ \\
\hline \multicolumn{2}{|c|}{ Time $\times$ bandage interaction effcet } & $F=8.649$ & $\mathrm{df}=2.397$ & $P<0.001$ \\
\hline \multicolumn{2}{|c|}{ Bandage effect (between-group effect) } & $F=4.106$ & $d f=1$ & $P=0.047$ \\
\hline
\end{tabular}




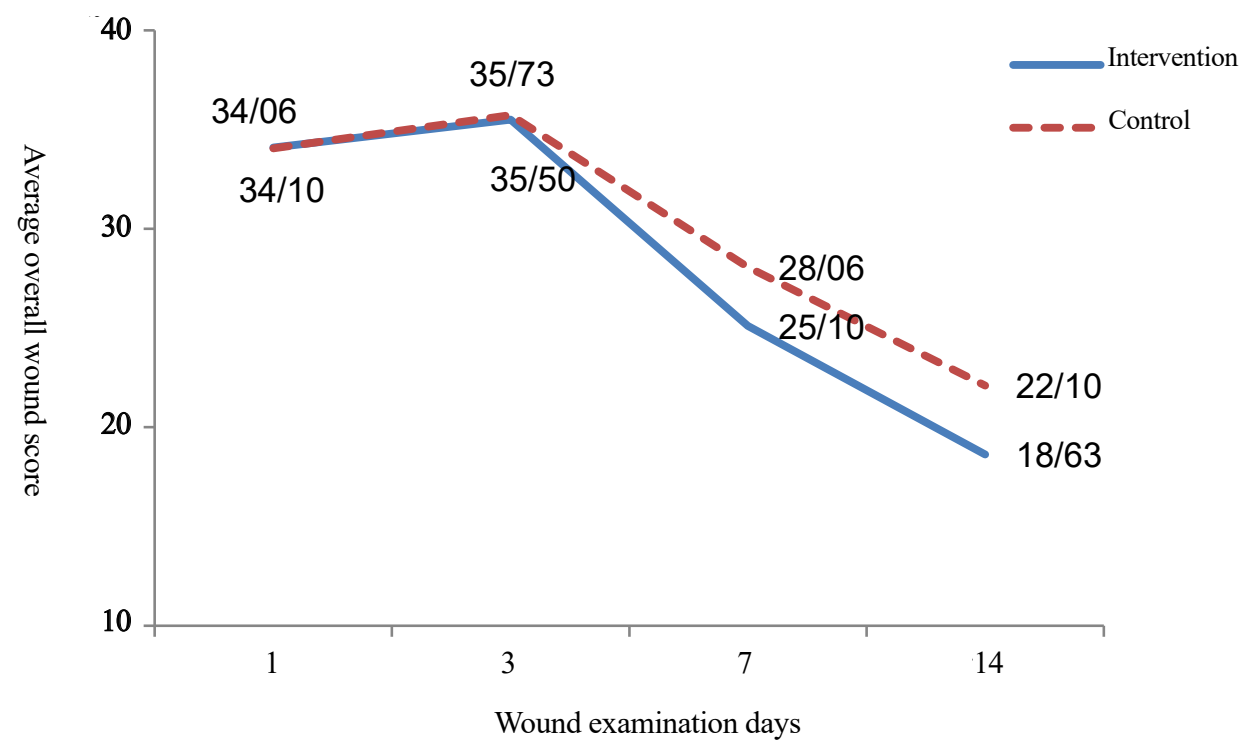

Figure 1. Comparing the final values of wound

significant in the intervention and control groups on the first, third, seventh, and fourteenth days $(\mathrm{P}=0.000, \mathrm{~F}=8.649)$. In other words, different days in wound healing were statistically significant in both research groups (Table 1).

The obtained findings respecting the effect of topical vitamin $\mathrm{C}$ solution on the healing of grade 2 burn wounds revealed that this solution caused more improvement in the intervention areas than in the control regions; thus, topical vitamin $\mathrm{C}$ was influential on wound healing.

\section{Discussion and Conclusion}

The current research results suggested that the rate of wound healing was significantly different between the study groups; topical vitamin C solution presented a greater effect on wound healing, compared to the control group.

The obtained results were consistent with those of similar studies conducted. We compared our data with those of the studies by Hadi Abbaspour and Mehdi Khaksari where they reported the impactful effects of kiwi on wound healing in rats, i.e., were in line with those of our results.

A study was conducted by Lima et al. on vitamin $\mathrm{C}$ for the healing of skin wounds in rats [18]; comparing these studies highlighted the improvement of granular tissue in the treatment with vitamin $\mathrm{C}$, i.e., consistent with the results of the above-mentioned study. Wesner and Gold entitled examined the impacts of topical vitamin $\mathrm{C}$ bed sores and chronic wounds; vitamin $\mathrm{C}$ solution could create fresh and abundant granular tissue in bedsores in patients [19].
Comparing this study with those of ours, we can point to the improvement of granular tissue at the site of receiving vitamin $\mathrm{C}$ solution.

Ulster et al. explored the effects of vitamin C on erythema after carbon dioxide laser [20]. Due to the greater effect of vitamin C solution in Ulster's study, the indication for using this substance was investigated in our research.

Our study data were inconsistent with those of Mehdi Poursadegh and associates. In this study, the effect of vitamin $\mathrm{C}$ was investigated on tympanic membrane graft repair in tympanoplasty [21]. In the above-mentioned study, using oral vitamin $\mathrm{C}$, the treatment process was studied at long intervals and only 5.2 months and 3 years later, i.e., one of the significant points of the study.

\section{Ethical Considerations}

\section{Compliance with ethical guidelines}

The present research was approved by the Graduate Council of Sabzevar School of Nursing and Midwifery (Ethics Code: IR.MEDSAB.REC.1394.60; IRCT Code: IRCT2015101224487N1).

\section{Funding}

The paper was extracted from the MSc. thesis of the corresponding author at the Department of Nursing, Faculty of Nursing and Midwifery, Sabzevar University of Medical Sciences, Sabzevar. 


\section{Authors' contributions}

Conceptualization and methodology: Hamid Robat Sarposhi; Data collection and writing - original draft: Mohammad Haddadi; Data analysis: Davood Movahedzadeh; Writing - review \& editing: Elahe Jaghor.

\section{Conflicts of interest}

The authors declared no conflicts of interest.

\section{Acknowledgements}

We would like to thank the Graduate Council of Sabzevar School of Nursing and Midwifery, the esteemed officials of Vasei Hospital, and the staff of the hospital's burn ward who contributed to this process. 
This Page Intentionally Left Blank 


\section{اثر ويتامين ث موضعي بر بهبود زخم سوختكًي}

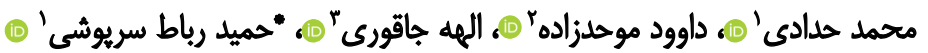

ا. كروه يرستارى، دانشكده يرستارى و مامايى، دانشكاه علوم يزشكى سبزوار، سبزوار، ايران.

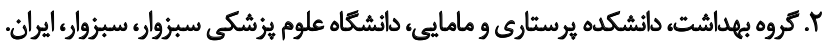

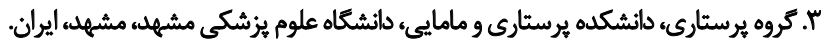

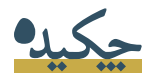

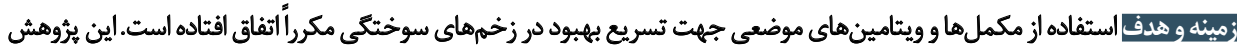

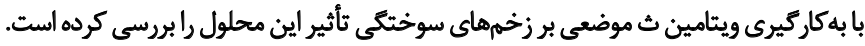

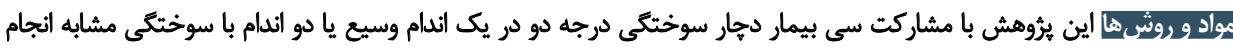

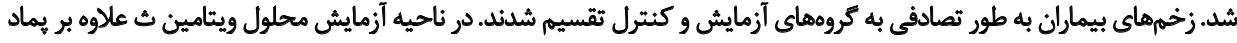

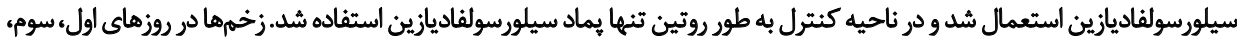

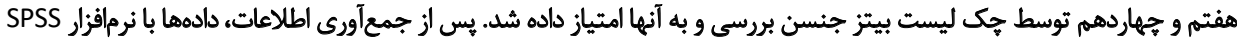

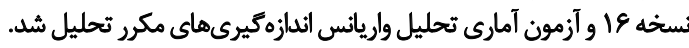

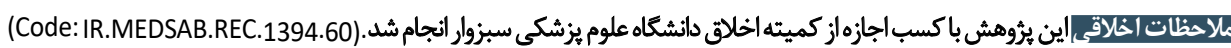

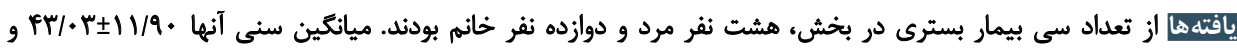

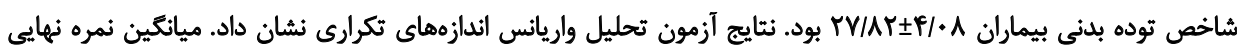

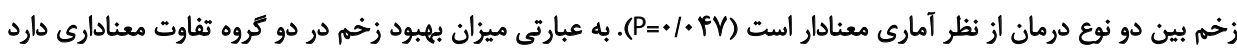

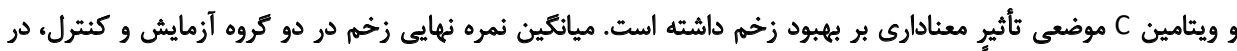

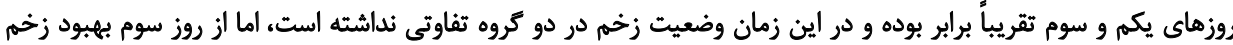

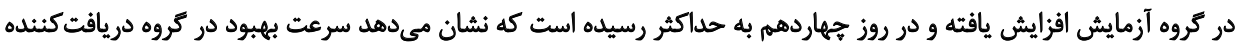

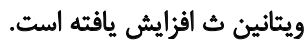
تتيجه كيرى سرعت بهبودى زخمم در كروه دريافت كنيده ويتانين ث افزايش يافته است.
\end{abstract}

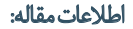

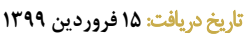

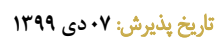

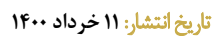

كائ1وا:

ويتامين ثه، بهيبوده

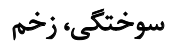

هزار نفر در بيمارستانها بسترى شده و بيش از ده هزار نفر بر بر بران

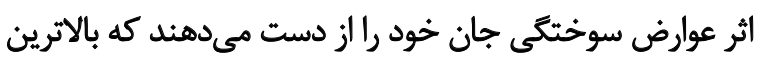

مقدمه

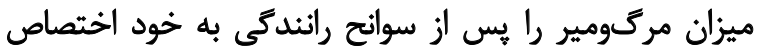
صدمات ناشى از تماس مستقيم يا برخورد با منابع كرمايي،

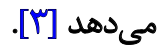

نسبت درصد مركى ناشى از سوختخى به كل مركها در در ايران

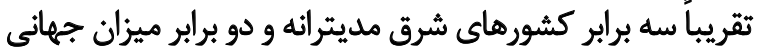

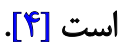

مديريت سوختكىها معمولاً روى مراقبت از بافت دجار

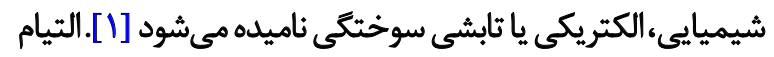

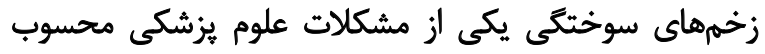

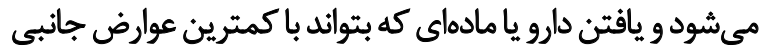

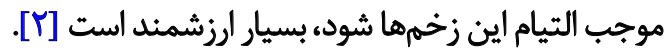

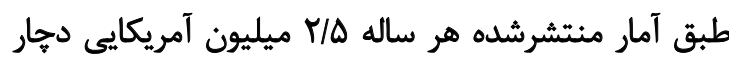

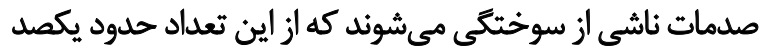

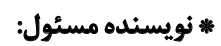

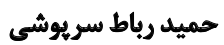

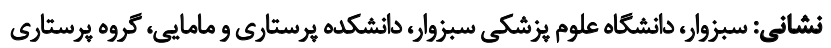

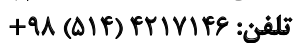

$$
\begin{aligned}
& \text { بست الكترونيكى: sarpooshi92@gmail.com }
\end{aligned}
$$




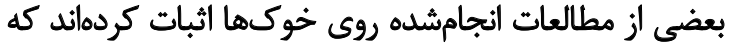

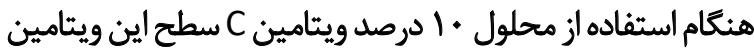

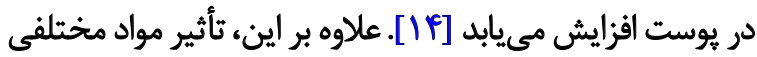

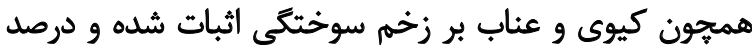

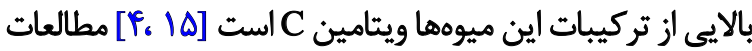

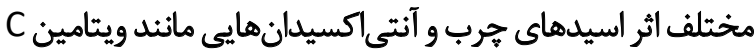

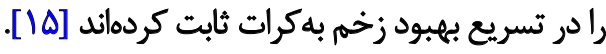
با توجه به شواهد بسيارى دال بر اثرات ويتامين C خوراكى

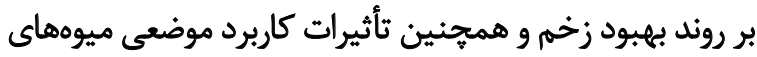

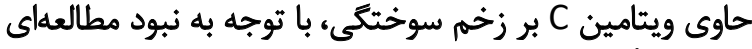

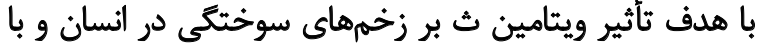

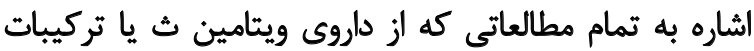

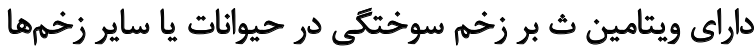

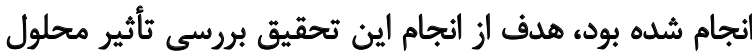

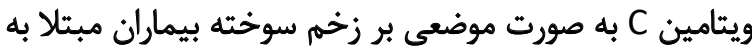

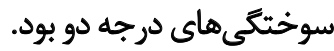

\section{مواد و روشها}

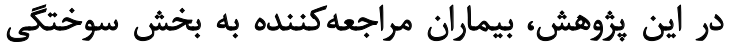

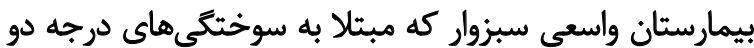

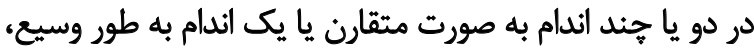

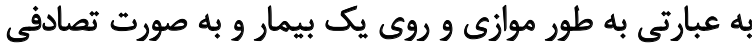

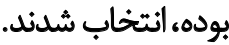

بر اساس حجم نمونه محاسبهشده، تعداد سى نفر واجد شرايط

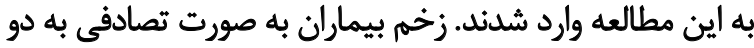

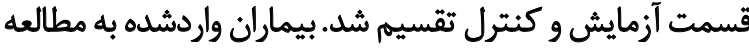

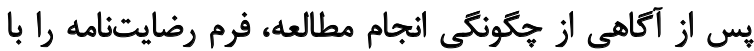

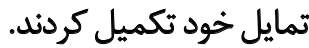

قبل از مطالعه زخم بيماران بر اساس جكيليست بيتز جنسن

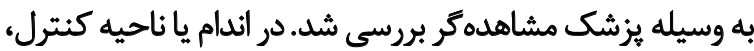

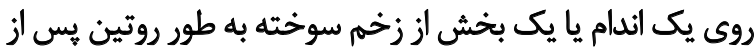

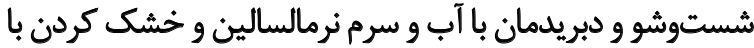

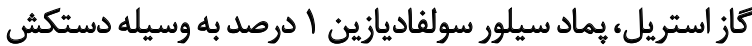
استريل به ضخامت ه/ ا ميلىمتر قرار داده شد. در اندام مقابل يا بخشى ديكر از اندام مورد مطالعه به عنوان إندان

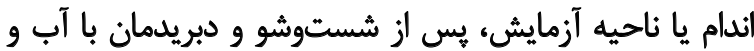

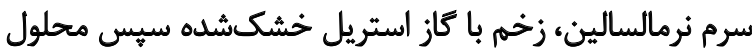

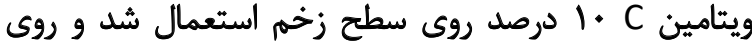

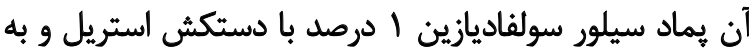

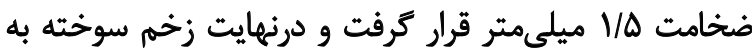

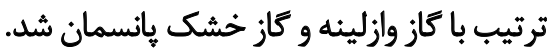

حداكثر سطحى از يك اندام كه با اندام متقارن مورد مقايسه
سوختكى متمركز مىشود.افزايش شناخت در مورد مكانيسمهاى بوني

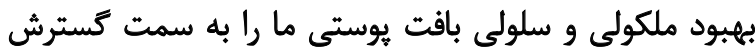

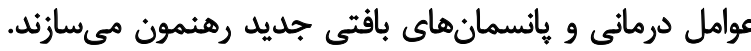

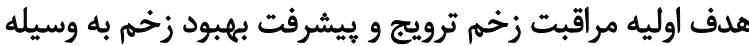

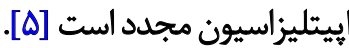

در حال حاضر براي بهبود زخم سوختكى از يانسمانهاي

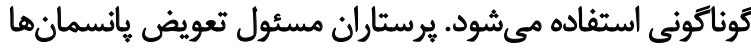

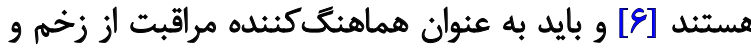

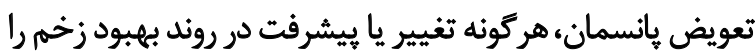

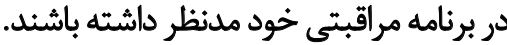

در سوختكىها ضمن تجمع ترشحات و بافتهائ سطح

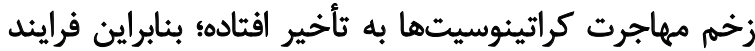

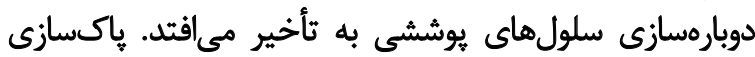

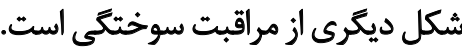

با توجه به اينكه بافت سوخته، بافتى غيرزنده و بلدون خونرساني

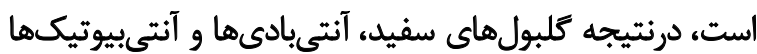

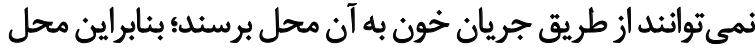

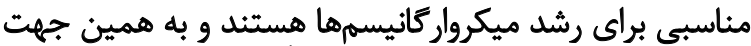

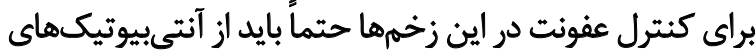

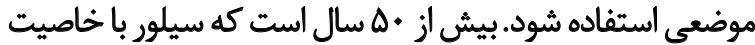

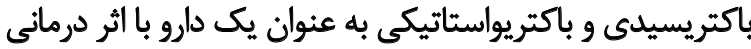

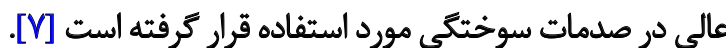

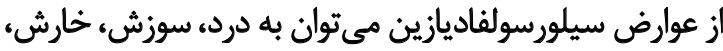

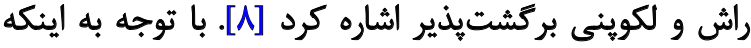

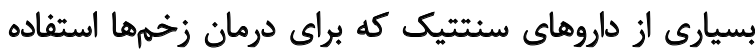

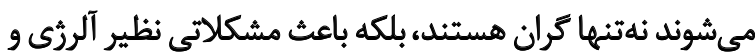

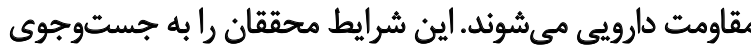

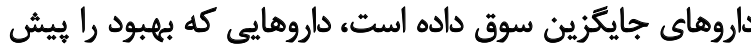

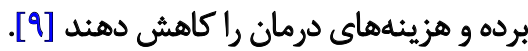
ويتامين Cمىتواند مكانيسم حفاظتى سيستم ايمنى را افزايش

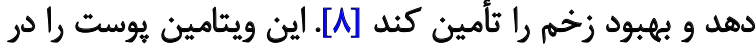

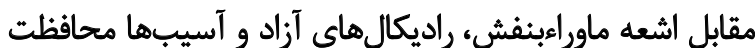

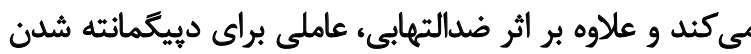

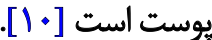

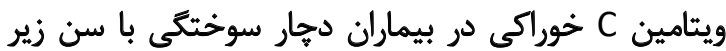

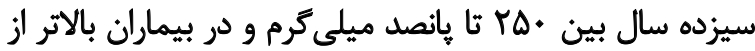

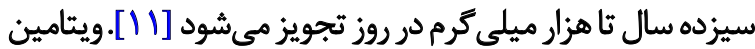

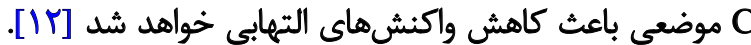

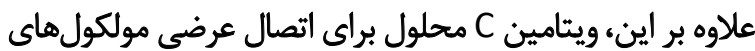

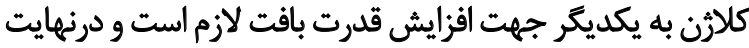

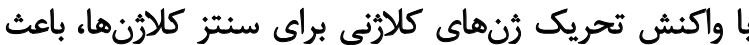

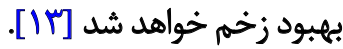




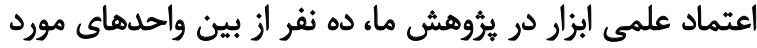

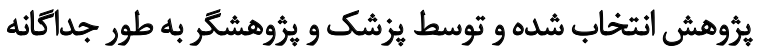

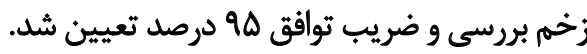
يُروهش با كسب اجازه از شوراى تحصيلات تكميلى دانشكده

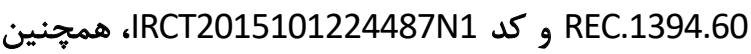
اخذ معرفى نامه و ارائه آن به مسئولين بيمارستان واسعى و بخش هئ سوختكى انجام شده است.

يس از جمع آورى اطلاعات، كدبندى انجام و دادهها وارد رايانه

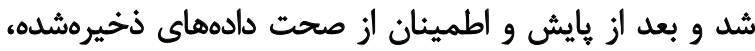

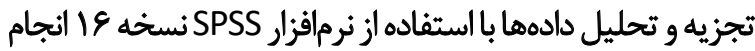

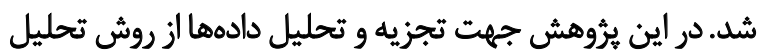
واريانس اندازههاى تكرارى استفاده شد.

يافتهها

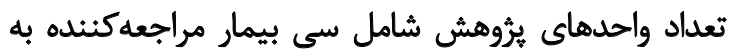

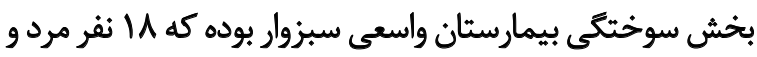

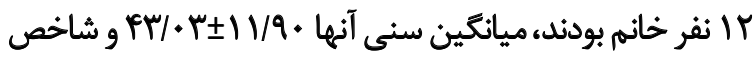

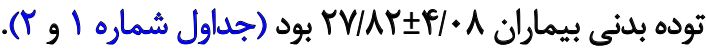
در راستاى هدف كلى بروهش، يعنى تعيين ثأثير محلول

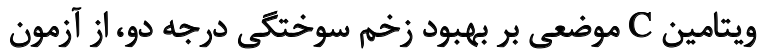

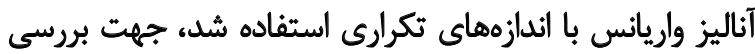

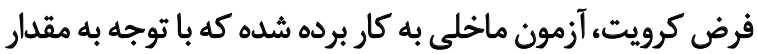

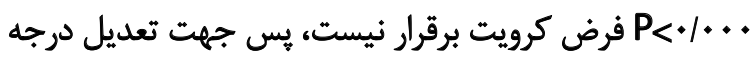

آزادى از مقدار إيسيلون كرين هاوس استفاده مي جشود.

نتايج آزمون تحليل واريانس اندازههاى تكرارى نشان داد

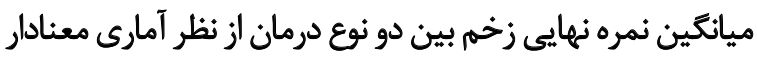

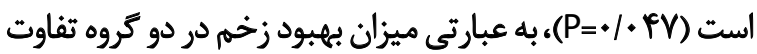

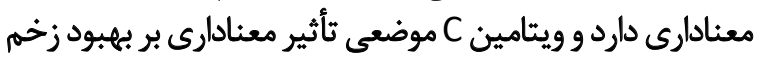
داشته است (جدول شماره ب).

در مورد اثرات درون كروهى نيز، اثر مدت زمان زمان بر ميانتين

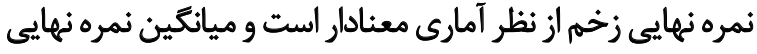

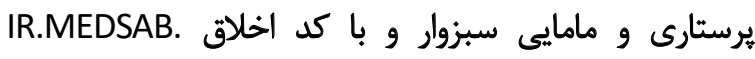

قرار مى كرفت يا يك بخش از يك عضو كه با بخش ديكر آن

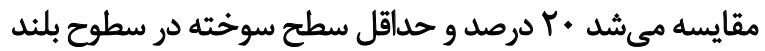

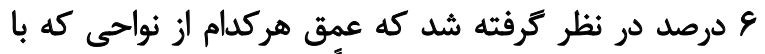

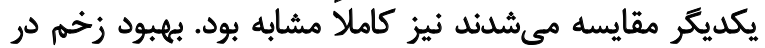

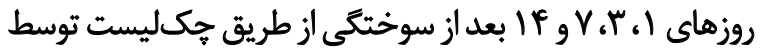

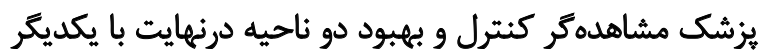
مقايسه شدند.

از معيارهاى ورود به مطالعه مي توان به موارد زير اشاره كرد:

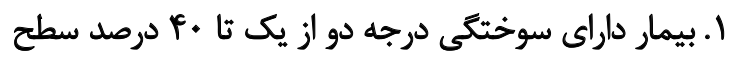

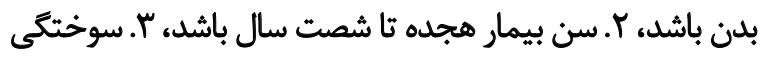

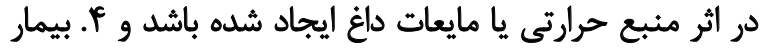

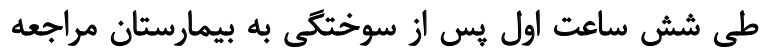

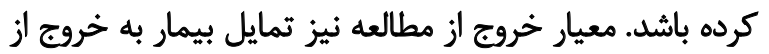
مطالعه است. باست.

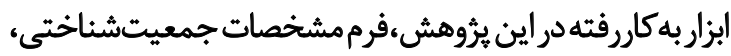

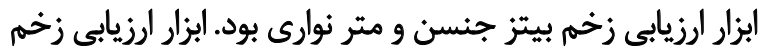

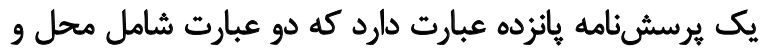

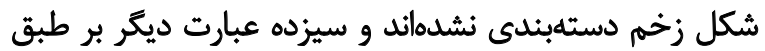

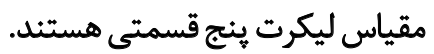

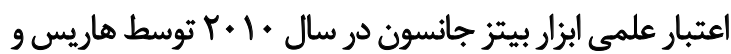

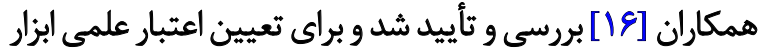

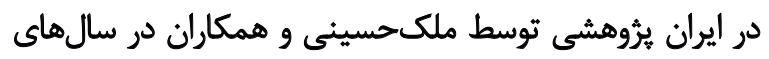

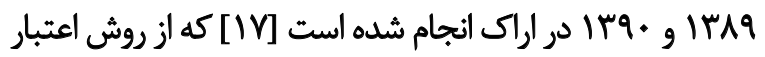
محتوا استفاده شد. در اين يثروهش اندكس اعتبار محتواى 19

درصد محاسبه شد.

براي تعيين اعتماد علمى ابزار توسط ملكحسيني و همكاران،

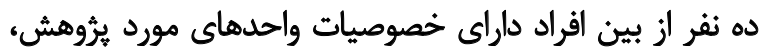

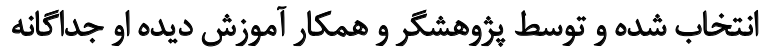

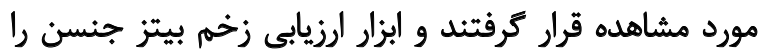
تكميل و ضريب همبستى رونى راتعيين كردند.

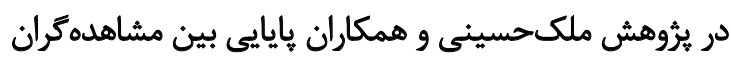
و ضريب كايا نيز براى هر آيتم سنجيده و وتأييد شد. براي تعيين مشانين

جدول ا. ويزّكى هاى فردى آزمودنى هاى مورد مطالعه در شاخصهاى كتمى

\begin{tabular}{|c|c|c|c|c|}
\hline ميانئين|انحراف معيار & حداكثر & حداقل & تعداد & مثغير \\
\hline$r r / . r \pm 11 / 9$. & $\Delta \vee \cdots$ & rV/.. & r. & سن \\
\hline rV/Arty/*A & $p+/ r^{2}$ & 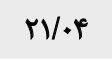 & r. & شاخص توده بلنى \\
\hline $99 / 4 \pm \pm T / 19$ & $10 \cdots+\cdots$ & $\Delta \cdots \cdots$ & r. & درأمد نمونهها \\
\hline$V / Q S \pm \Delta / g r$ & re & 1 & $r$ & درصد سوختكى \\
\hline
\end{tabular}


جدول r. ويرّكى هاي فردى آزمودنى هاى مورد مطالعه در شاخصهاي كيفى

\begin{tabular}{|c|c|c|}
\hline فواوانى (درصد) & نوع & متغير \\
\hline$M(\varepsilon \cdot)$ & مرد & \multirow[b]{2}{*}{ جنس } \\
\hline$M\left(r_{*}\right)$ & زن & \\
\hline$r \cdot(89 \mid 89)$ & حرارتى & \multirow{2}{*}{ عامل سوختكى } \\
\hline 1. ( $(\pi / / \pi T)$ & مايعات داغ & \\
\hline 11 (rg/\&9) & ابتدائى و كمتر & \multirow{4}{*}{ سطح سواد } \\
\hline$\theta(r)$ & سيكل & \\
\hline$q\left(r_{0}\right)$ & دييلم & \\
\hline$V(\pi / M / M)$ & ليسائس & \\
\hline
\end{tabular}

\section{这}

جدول سا. ميانكين و انحراف معيار نمره نهايى زخهم در دو كروه آزمايش و كنترل بعد از بانسمان

\begin{tabular}{|c|c|c|c|}
\hline \multicolumn{3}{|c|}{ ميانغيندانحراف معيار } & \multirow{2}{*}{ شاخم آمارى } \\
\hline كثترل & & آزآمايش & \\
\hline$M \in / .9 \pm 1 / m$ & & $\mu \in / 1 . \pm 1 / \mu$ & روز اول \\
\hline$r \Delta / M^{\mu} \pm \psi / N^{\mu}$ & & $r \Delta / \Delta+ \pm \psi / \nu^{\mu}$ & روز سوم \\
\hline$r N \cdot G \pm Y / q_{.}$ & & $r \Delta / 1+ \pm r / \wedge q$ & روز هفتم \\
\hline$M T / 1+ \pm F / F$ & & WEYTY/IF & روز جهاردهم \\
\hline $\mathrm{P}<+1 \ldots 1$ & 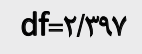 & $F=\Delta r \Delta / / \Delta C$ & اثر زمان (اثر درون كروهى) \\
\hline$P<\cdot 1 \ldots 1$ & $d f=r / \% q r$ & $F=N$ Peq & اثر تعاملى يانسمان و زمان \\
\hline $\mathrm{P}=. / . e \mathrm{~V}$ & $d f=1$ & $F=F / 1.8$ & اثر يانسمان (اثر بين كروهي) \\
\hline
\end{tabular}

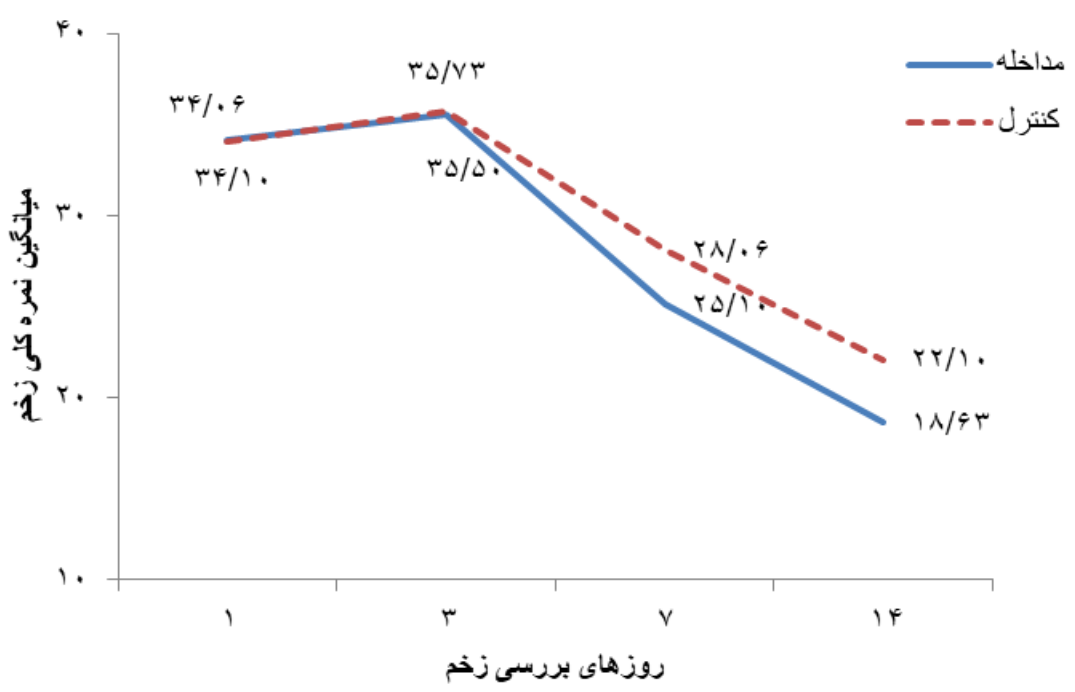


عروق جديد بيشتر نسبت به گروه كنترل مشاهده شد [1/]، در

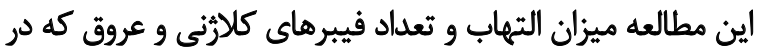

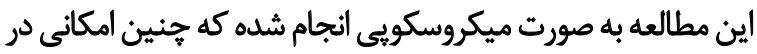

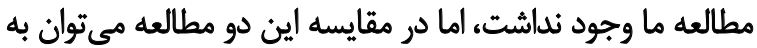

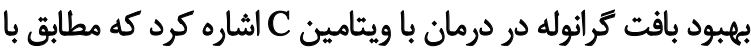

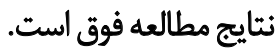

در مطالعه انجامشده توسط وسنرو گملدبا عنوان درمان زخم بستر

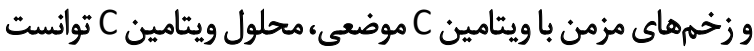

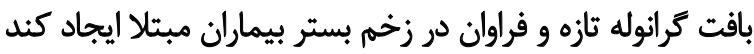

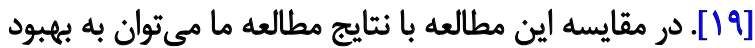
بافت ترانوله در محل دريافت كنينده محلول ويتامين C اشاره كرد.

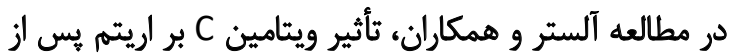

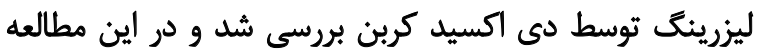

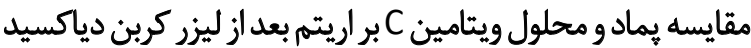

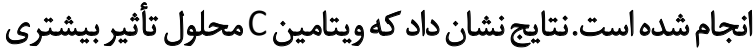

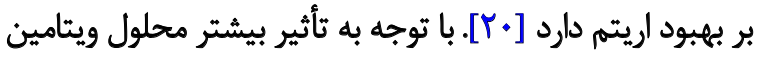

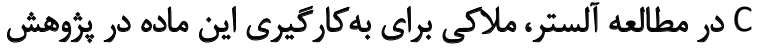

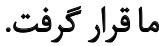

مطالعه ما با مطالعه مهلى يورصادق و همكاران همراستا نبود،

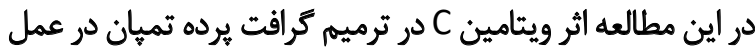

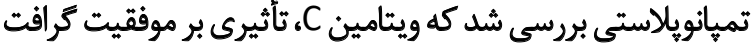

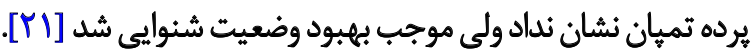
در مطالعه فوق با بهكاركيرى ويتامين C حوراكى، روند درمان

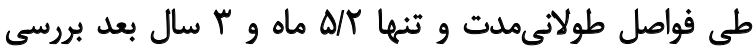

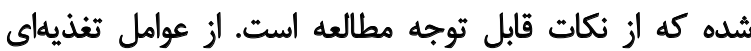

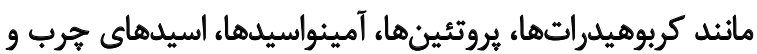

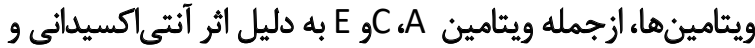

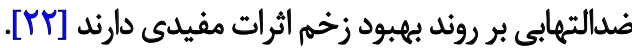
ويتامين C به تنهايى باعث كاهش نياز به احياى مايعات در

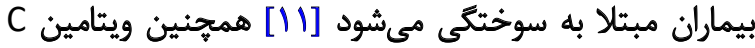
موضعى باعث كاهش واكنشهاى التهابى خواهد شد [1 II]].

\section{مالاحظات اخلاقى}

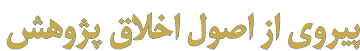

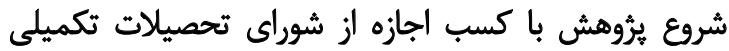

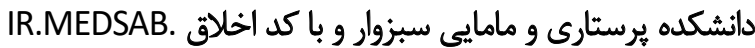

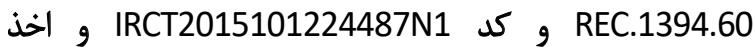
معرفىنامه وارائه آن به مسئولين بيمارستان واسعى سبزوار و بخش واخش سوختكى انجام شده است.

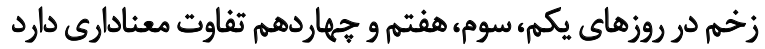

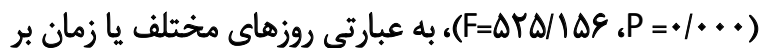
بهبود زخم تأثير كذار بوده است.

براى بررسى اثر تعاملى نوع هانسمان با زمان در آزمون تحليل

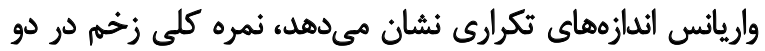

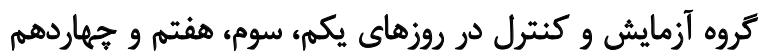

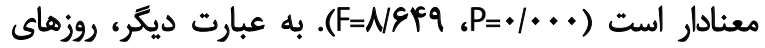
هختلف در بهبود زخم در دو تروه به لحاظ آمارى معنادار بودهد

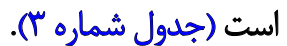

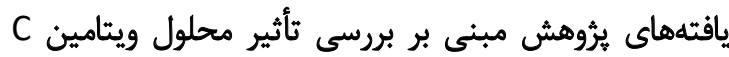

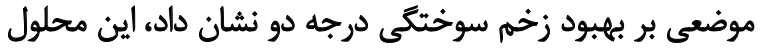

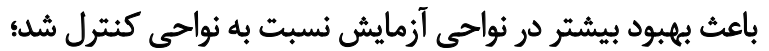

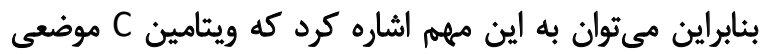

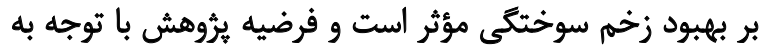
يافتههاى يثوهش مورد تأييد است.

در راستاي هدف كلى برؤهش، يعنى تعيين تأثير محلول

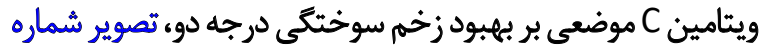

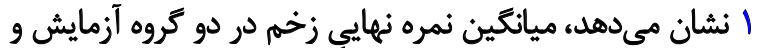

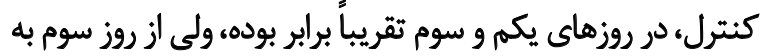

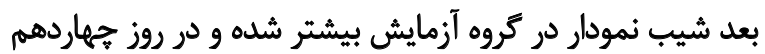

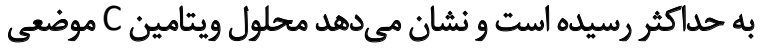
ثأثير بيشترى بر بهبود زخم نسبت به كروه كنترل داشته است.

\section{نتيجنهي}

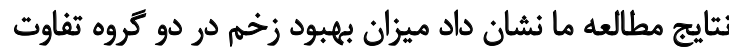

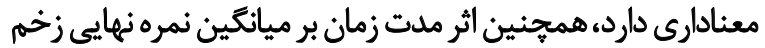

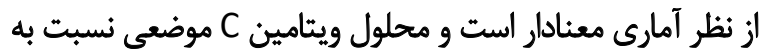
كروه كنترل تأثير بيشترى در بهبود زخمه داشته است است.

نتايج بهدستآمده با نتايج مطالعات انجام شده در اين زمينه

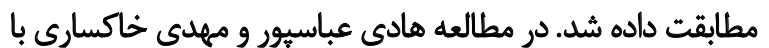

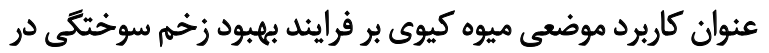

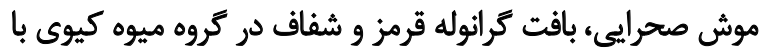

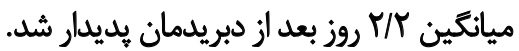
استفاده از ميوه كيوى در جداسازى بافتهاى نكروزه سريعتر از

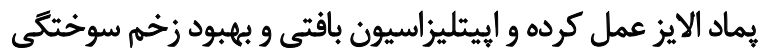

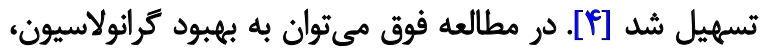

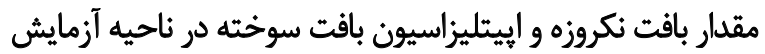
اشاره كرد كه مى توان آن را همروراستا با با مطالعه ما دانست.

در مطالعه انجامشده توسط ليماو همكاران با عنوان ويتامين

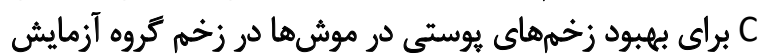

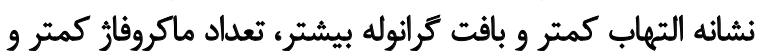




$$
\text { مام }
$$

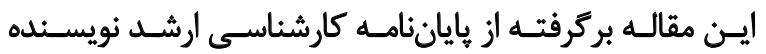

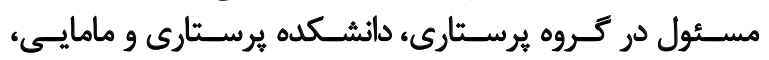

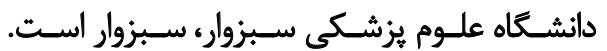

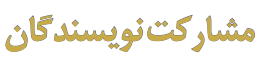

مفهوم سازى و روششناسى: حميد رباط سريوشى؛ تردآدرى و و

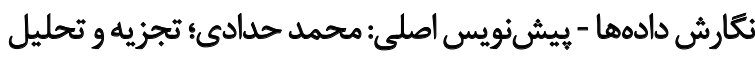

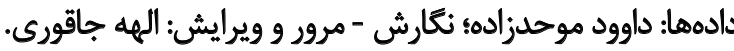

$$
\text { ethos gles }
$$

بنابر اظهار نويسندكان اين مقاله تعارض منافع ندارد.

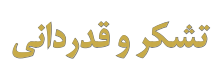

از شوراى تحصيلات تكميلى دانشكده يرستارى و مامايى ماريى

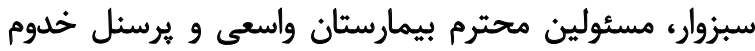

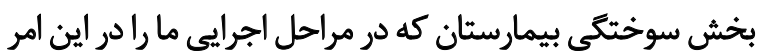
مساعدت كردند، تشكر و قدردانى مي كنيميم. 


\section{Refrences}

[1] Black JM, Hawks JH. Medical surgical nursing: Clinical management for positive outcomes, 8e (2 Vol Set) without CD. Mumbai: Elsevier India; 2009. https://www.elsevier.com/books/medical-surgical-nursingclinical-management-for-positive-outcomes-8e-2-vol-set-without-cd/ black/978-81-312-2982-8

[2] Behrouz B, B. Hashemi F. [Protective efficacy of a divalent candidate vaccine consisting of type a flagellin and pilin against dermal pseudomonas aeruginosa infection in a murine burn model (Persian)]. Iran J Med Microbiol. 2018; 12(4):248-59. http://ijmm.ir/article-1-819-fa.html

[3] Daryabeigi R, Heidari M, Hosseini SA, Omranifar M (2010)Comparison of healing time of the 2 degree burn wounds with two dressing methods of fundermol herbal ointment and $1 \%$ silver sulfadiazine cream. Iran J Nurs Midwifery Res. 2010; 15(3):97-101. [PMID][PMCID]

[4] Abbaspour H, Khaksari M. [Topical effectiveness of kiwifruit on burn wound healing in male rats (Persian)]. J North Khorasan Unive Med Sci. 2013; 5(1):91-7. [DOI:10.29252/jnkums.5.1.91]

[5] Bosworth-Bousfield C. Burn trauma: Management and nursing care. London: Wiley; 2001. https://books.google.com/ books?id=C7Z7QgAACAAJ\&dq

[6] Lynn P. Taylor's clinical nursing skills: A nursing process approach. Philadelphia: Lippincott Williams \& Wilkins; 2018. https://www.amazon. com/Taylors-Clinical-Nursing-Skills-Approach/dp/1451192711

[7] O'Connell Smeltzer SC, Bare BG, Hinkle JL, Cheever KH. Brunner and Suddarth's Text book of medical surgical nursing. Philadelphia: Lippincott Williams \& Wilkins; 2010. https://books.google.com/ books?id=SmtjSD $1 \times 688 \mathrm{C} \& \mathrm{dq}$

[8] Abgoon M. [Iranian generic medicine accompanying interrogation measures (Persian)]. $3^{\text {rd }}$ ed. Tehran: Noore Danesh; 2000. http://opac. nlai.ir/opac-prod/bibliographic/627137

[9] Heck E, Head M, Nowak D, Helm P, Baxter C. Aloe vera (gel) cream as a topical treatment for outpatient burns. Burns. 1981; 7(4):291-4. [DOI:10.1016/0305-4179(81)90112-1]

[10] Telang PS. Vitamin C in dermatology. Indian Dermatol Online J. 2013; 4(2):143-6. [DOI:10.4103/2229-5178.110593][PMID][PMCID]

[11] Herndon DN. Total burn care. New York: Saunders Elsevier; 2007. https://books.google.com/books?id=m_QnStA_JPsC\&dq

[12] Singh P. Role of topical ascorbic acid in management of refractory corneal ulcer. IOSR J Pharm. 2012; 2(6):1-4. [DOI:10.9790/3013-265014]

[13] Pinnell SR, Modey DL. The benefits of topical vitamin C (L-ascorbic acid) for skin Careano UV protection. J Appl Cosmetol. 1999; 18:126-34. https://www.semanticscholar.org/paper/THE-BENEFITS-OF-TOPICALVITAMIN-C-(L-ASCORBIC-ACID)-Pinnell-Madey/f71a1d1f09785ed075611c2276e79c48bed3b6db

[14] Draelos ZD. Nutrition and enhancing youthful-appearing skin. Clin Dermatol. 2010; 28(4):400-8. [DOI:10.1016/j.clindermatol.2010.03.019] [PMID]

[15] Ashrafi K, Esmaeli E, Shahinfard N, Ansari R, Parvin N, Namjoo A, et al . [The effect of hydroalcoholic extracts of Zizipus vulgaris $L$. on burn healing (Persian)]. J Shahrekord Univ Med Sci. 2011; 12(4):78-82. http://78.39.35.44/article-1-401-en.html

[16] Harris C, Bates-Jensen B, Parslow N, Raizman R, Singh M, Ketchen R. Bates-Jensen wound assessment tool: Pictorial guide validation project. J Wound Ostomy Continence Nurs. 2010; 37(3):253-9. [DOI:10.1097/ WON.0b013e3181d73aab][PMID]
[17] Malekhosseini A, Ghaffarzadegan R, Alizadeh SA, Ghafarzadegan R, Haji Agaei R, Ahmadlou M. [Effect of aloe vera gel, compared to $1 \%$ silver sulfadiazine cream on second-degree burn wound healing (Persian)]. Complement Med J. 2013; 3(1):67-78. https://www.sid.ir/fa/journal/ ViewPaper.aspx?ID=201815

[18] Lima CC, Pereira APC, Silva JRF, Oliveira LS, Resck M, Grechi CO, et al. Ascorbic acid for the healing of skin wounds in rats. Braz J Biol. 2009; 69(4):1195-201. [DOI:10.1590/S1519-69842009000500026]

[19] Mester AJ. Treatment of bedsores and chronic ulcers with topical ascorbic acid. Lancet. 1961; 277(7172):343-4. [DOI:10.1016/S0140 6736(61)91521-5]

[20] Alster TS, West TB. Effect of topical vitamin C on postoperative carbon dioxide laser resurfacing erythema. Dermatol Surg. 1998; 24(3):331-4. [DOI:10.1111/j.1524-4725.1998.tb04163.x][PMID]

[21] PourSadegh M, Seyyedi M, Mohammadi A. [Evaluation of the effects of Vitamin C on tympanic graft repair in tympanoplasty (Persian)]. J Birjand Univ Med Sci. 2006; 13(3):9-15. http://journal.bums.ac.ir/article-1-99-en.htm

[22] Arnold M, Barbul A. Nutrition and wound healing. Plast Reconstr Surg. 2006; 117(7 Suppl):42S-58S. [DOI:10.1097/01. prs.0000225432.17501.6c][PMID] 